\title{
Experimental and numerical study of thermal fatigue behavior under variable amplitude induced by pulsed laser
}

\author{
SiNing Pan ${ }^{1,2}$, Gang $\mathrm{Yu}^{1,2^{*}}$, XiuLi He ${ }^{1,2^{*}}$, ShaoXia $\mathrm{Li}^{1}$, and $\mathrm{Ru} \mathrm{Chen}^{1,2}$ \\ ${ }^{1}$ Institute of Mechanics, Chinese Academy of Sciences, Beijing 100190, China; \\ ${ }^{2}$ School of Engineering Science, University of Chinese Academy of Sciences, Beijing 100049, China
}

Received February 13, 2018; accepted March 12, 2018; published online May 23, 2018

Citation: S. N. Pan, G. Yu, X. L. He, S. X. Li, and R. Chen, Experimental and numerical study of thermal fatigue behavior under variable amplitude induced by pulsed laser, Sci. China-Phys. Mech. Astron. 61, 104621 (2018), https://doi.org/10.1007/s11433-018-9201-0

Thermal fatigue is the typical failure mode of high-temperature combustion chamber components used in power plants and in the aerospace and transportation industries [1-3]. With growing demand for good performance and high reliability, thermal fatigue is becoming a serious problem. The thermal working conditions of combustion chamber components include start-stop cycles and routine working cycles simultaneously $[4,5]$. The former refer to large temperature changes, with the amplitude of $200^{\circ} \mathrm{C}-500^{\circ} \mathrm{C}$ and time period of several hundred seconds that are responsible for low-cycle fatigue (LCF) damage. The latter correspond to low temperature amplitudes of $20^{\circ} \mathrm{C}-60^{\circ} \mathrm{C}$, with time period of milliseconds, and are responsible for high-cycle fatigue (HCF) damages. The LCF or HCF tests belong to constantamplitude cyclic loading. In contrast, the loading combination of LCF and HCF leads to combined-cycle fatigue (CCF) because of the variable amplitude cyclic loading. Considering the interaction of thermal loadings under different amplitudes, the thermal fatigue of CCF tests is expected to differ from that of LCF or HCF tests [6].

Several heat sources have been used in thermal fatigue tests, such as high-frequency induction [7], quartz lamps [8], muffle furnaces [9], and continuous wave lasers [10,11]. However, owing to the intrinsic limitations of the above

*Corresponding authors (Gang Yu, email: gyu@imech.ac.cn; XiuLi He, email: xlhe@imech.ac.cn) methods, the superimposed HCF thermal loading within milliseconds is difficult to achieve. Therefore, owing to the high temporal and spatial controllability, the pulsed laser is the ideal heat source for thermal CCF test $[2,12,13]$, if appropriate laser parameters are selected.

Thermal fatigue induced by pulsed laser has been well studied. Zhu and Miller [1] paid attention to thermal barrier coating systems under CCF thermal conditions in diesel engine applications and investigated the initiation and propagation of fatigue cracks. Long and Zhou [14] studied thermal fatigue in particle-reinforced metal-matrix composites induced by pulsed laser heating and mechanical loading and simulated the temperature and macroscopic and microscopic stress. During the CCF thermal cycles induced by pulsed laser, surface cracks initiate and propagate gradually. This time-dependent change affects the absorption of input energy, thus affecting the thermal deformation [15]. However, the pulsed laser parameters are kept constant in most studies of CCF thermal loading, resulting in instability and uncontrollability. Furthermore, stresses generated by pulsed lasers can initiate surface cracks, but the details of crack evolution under the complex LCF and HCF conditions are not well understood, particularly for the cast iron materials used in combustion chambers.

In this study, we investigated GJV-450 (compacted graphite) cast iron, which is typically used in the cylinder heads of diesel engines. The vermicular graphite has an average 
length of $70-100 \mu \mathrm{m}$ and width of $10 \mu \mathrm{m}$. The specimens were cut into cylindrical $\Phi 18 \times 5 \mathrm{~mm}$ pieces.

The experiments were performed using an in-house testing apparatus [16] (Figure 1(a)). A Nd:YAG millisecond pulsed laser with wavelength of $1.064 \mu \mathrm{m}$ was the heat source. The pulse width and the diameter of the laser were constant at $10 \mathrm{~ms}$ and $6 \mathrm{~mm}$, respectively. To produce the variable amplitude thermal cycles, a complete testing cycle was designed to include three stages: the heating stage, the high-cycle stage, and the cooling stage, as shown in Figure 1(b). The first stage was controlled using a PC with a temperature control module and the maximal temperature was set as $450^{\circ} \mathrm{C}$. A high repetition rate of $20 \mathrm{~Hz}$ and low pulse energy of $9 \mathrm{~J}$ was used in the first stage. After reaching the maximal temperature, the second stage continued in time-controlled mode and the heating time was $250 \mathrm{~s}$. At the same time, the laser parameters were switched to lower repetition rate $(4 \mathrm{~Hz})$ and higher pulse energy $(15 \mathrm{~J})$ to induce small-amplitude cycles. Once the first two stages were completed, the pulsed laser was turned off; then, compressed air was blown to the backside of the specimen until the detected temperature dropped to $100^{\circ} \mathrm{C}$.

A two-dimensional axisymmetric model for the longitudinal sections was proposed to describe the thermal and structural coupling under variable amplitude thermal loading. The heat conduction, convection, and radiation effects were considered in the transient finite element analysis. During pulsed laser heating, the surfaces were under natural convection with a heat exchange coefficient of $20 \mathrm{~W} \mathrm{~m}^{-2} \mathrm{~K}^{-1}$. After the pulsed laser application, the back surface was under forced convection with a heat exchange coefficient of $100 \mathrm{~W} \mathrm{~m}^{-2} \mathrm{~K}^{-1}$. With regard to the significant temperature change on the specimen surface and high thermal gradient across its thickness, the mesh size within 1-mm depth from the surface decreased. The number of meshes was 28350. The different crack parameters induced by pulsed laser heating were considered in the geometric model, in which the cracks with the same width and depth were considered. The material was assumed as homogenous, and the material parameters are listed in Table 1.

The temperature distribution is described by the Fourier heat conduction equation [17], and the boundary conditions on the top surface (including the cracked zone and the uncracked zone) during the pulse duration are as follows:

If $(i-1) \cdot(w+v)+w<x<i \cdot(w+v)$, and $i=1 \sim n$, then

$-\left.k \frac{\partial T}{\partial y}\right|_{y=0}=\alpha(T) I(x)$.

If $(i-1) \cdot(w+v) \leq x \leq(i-1) \cdot(w+v)+w$, and $i=1 \sim n$, then

$-\left.k \frac{\partial T}{\partial y}\right|_{y=b}=\alpha(T) I(x)$,

where $\alpha(T)$ is the laser absorptivity, which depends on the

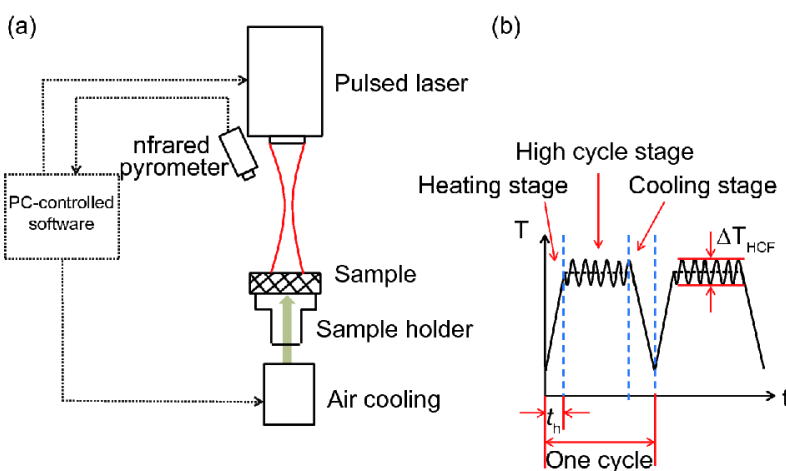

Figure 1 (Color online) (a) Experimental setup and (b) testing cycle.

Table 1 Properties of GJV-450

\begin{tabular}{|c|c|c|c|c|c|c|}
\hline $\begin{array}{c}T \\
(\mathrm{~K})\end{array}$ & $\begin{array}{c}K \\
\left(\mathrm{~W} \mathrm{~m}^{-2} \mathrm{~K}^{-1}\right)\end{array}$ & $\begin{array}{c}C \\
(\mathrm{~J} / \mathrm{kg} \cdot \mathrm{K})\end{array}$ & $\begin{array}{c}\rho \\
\left(\mathrm{kg} / \mathrm{m}^{3}\right)\end{array}$ & $v$ & $\begin{array}{c}\alpha \\
\left(\mathrm{K}^{-1}\right)\end{array}$ & $\begin{array}{c}E \\
(\mathrm{GPa})\end{array}$ \\
\hline 293 & 38.0 & 470 & \multirow{4}{*}{7079} & \multirow{4}{*}{0.25} & - & 156 \\
\hline 373 & 34.9 & 500 & & & $11.7 \times 10^{-6}$ & 152 \\
\hline 673 & 31.6 & 595 & & & $13.3 \times 10^{-6}$ & 148 \\
\hline 823 & 29.5 & 675 & & & $14.2 \times 10^{-6}$ & 90 \\
\hline
\end{tabular}

material and temperature, and $n, w, v$, and $b$ are the crack number, crack width, crack interval, and crack depth, respectively. The super-Gaussian spatial distribution of the pulsed laser is as follows:

$I(x)=I_{0} \exp \left[-2\left(\frac{x}{R}\right)^{N}\right]=\frac{E}{\pi R^{2} \tau} \exp \left[-2\left(\frac{x}{R}\right)^{9}\right]$,

where $R, E$, and $\tau$ are the specimen radius, the single-pulse energy, and pulse duration, respectively. $N$ is the order of the super-Gaussian function and is set as 9 in the experiment.

Stress and strain analysis is conducted using the temperature field results for loading. The model is free of constraints, and the symmetrical displacement boundary condition is applied to the symmetric axis.

There are two important thermal parameters reflecting the combined thermal process, i.e., the heating time $\left(t_{\mathrm{h}}\right)$ in the heating stage and the temperature range in the high-cycle stage $\left(\Delta T_{\mathrm{HCF}}\right)$, which are closely related to the crack propagation on the substrate surface. During the tests, the nonlinear change in absorptivity is significant, particularly after the surface cracks are initiated within the irradiated region. There are multiple reflections of the laser beam in the grooves of the cracked surfaces. With more and deeper cracks, more energy is absorbed, thereby making the heating stage shortened and the temperature range increased. The above physical effects are considered in the proposed numerical model.

Based on the above analysis, the temperature histories for different crack parameters are simulated and compared with the experimental results. The measured temperature of the 
40th cycle is shown in Figure 2(a). The surface temperature changes from $100^{\circ} \mathrm{C}$ to $450^{\circ} \mathrm{C}$ in $8 \mathrm{~s}$, and the high-cycle stage begins. Therefore, a temperature oscillation around $450^{\circ} \mathrm{C}$ is inherently superimposed on the existing cycles. From the insert graph in Figure 2(a), $\Delta T_{\mathrm{HCF}}$ is about $60^{\circ} \mathrm{C}$. The calculated temperature is shown in Figure 2(b), with ten, 200$\mu \mathrm{m}$-deep cracks. The simulated results and measurements agree, and the simulated $\Delta T_{\mathrm{HCF}}$ is $60^{\circ} \mathrm{C}$.

To compare the model and experimental crack parameters, the crack depth after different cycles is observed, as shown in Figure 3. From Figure 3(a), cracks with depth of about $50 \mu \mathrm{m}$ are denoted by the arrows and are tested after two cycles. During the cycles, the cracks propagate continually along the thickness direction. The depth reached about $150 \mu \mathrm{m}$ after 20 cycles, as shown in Figure 3(b). As the oxidation proceeds gradually, oxygen diffuses inward and deepens the cracks. After 40 cycles, a crack with nearly $200 \mu \mathrm{m}$ depth is observed (Figure 3(c)), which is almost similar to the value used in the simulation.

Owing to the limited heat effect depth of the pulsed laser heating, the induced crack depth strongly depends on the temperature distribution within the substrate, particularly in the thickness direction. When pulsed laser heating is applied, high thermal gradient is induced along the thickness direction. The simulated results suggest that the transient tem-
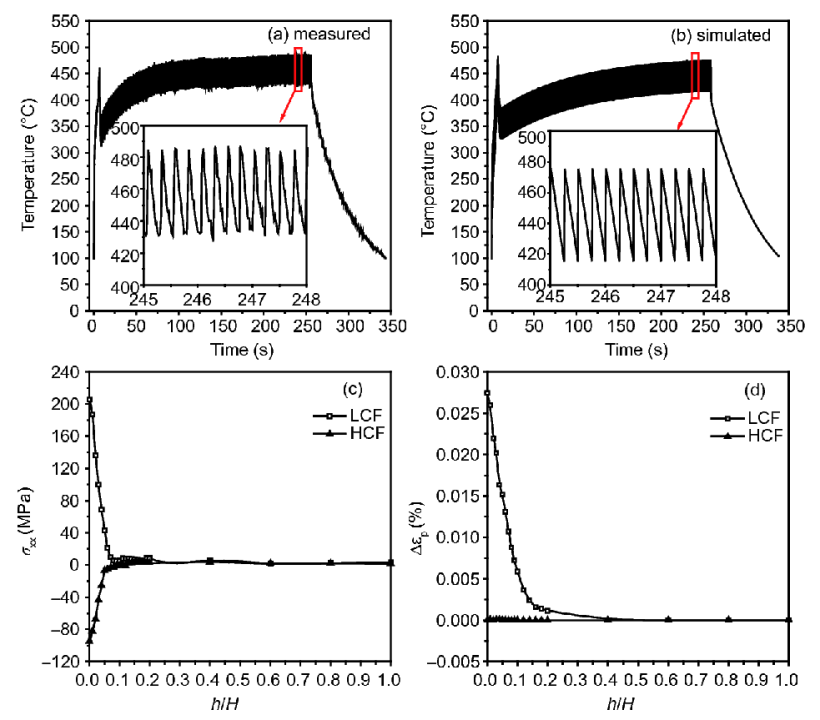

Figure 2 (Color online) (a) Measured temperature histories; (b) simulated temperature histories; (c) simulated stress along depth; (d) simulated plastic strain range along depth.

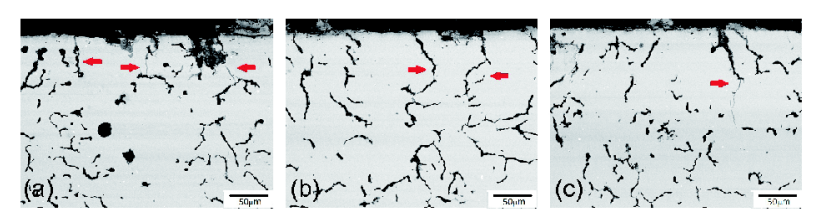

Figure 3 (Color online) Cross section of the specimen in the irradiated center (SEM): (a) after two cycles; (b) after 20 cycles; (c) after 40 cycles. perature change occurs only within a thin layer below the surface. This layer is defined as the interaction depth at which obvious temperature discrepancies occur [18] and strongly depends on the thermal boundary conditions. The temperature gradient owing to the LCF thermal loading is higher than the one owing to the HCF thermal loading, thus producing a larger thermal interaction depth.

The unsteady heat transfer is characterized by the dimensionless Fourier number $\left(F o=\delta D / l^{2}\right)$, which compares a characteristic body with dimension 1 and an approximate temperature-wave penetration depth for a given time $\delta$. The model used in this study is simplified as an infinite plate with a certain thickness, i.e., $l$ is equal to half the specimen thickness. $\delta$ is generally defined as the interval of each pulse, e.g., $0.05 \mathrm{~s}$ at $20 \mathrm{~Hz}$ and $0.25 \mathrm{~s}$ at $4 \mathrm{~Hz} . D$ is the thermal diffusivity of the material and its value is $7.06 \times 10^{-6} \mathrm{~m}^{2} / \mathrm{s}$ for GJV-450. Therefore, the $F o$ number for the heating stage and the high-cycle stage is 0.056 and 0.28 , respectively. According to ref. [19], when the Fo number of an infinite plate is $>0.24$, the effect of the initial conditions disappears, entering into the regular regime of unsteady heat transfer. For pulsed laser heating, the Fo number strongly depends on the pulse interval. Considering the above analysis, $4 \mathrm{~Hz}$ is the critical repetition rate for inducing stable temperature oscillations because once the pulse of $4 \mathrm{~Hz}$ is removed, the temperature difference along the thickness direction decreases rapidly and reaches quasi-uniform state. If the pulse repetition rate is increased to $20 \mathrm{~Hz}$, i.e., the Fo number is reduced to 0.056 , the temperature gradient along the thickness is high after the pulse removal. Once the next pulse acts, the temperature difference keeps increasing, resulting in significant thermal accumulation.

As mentioned above, frequent temperature changes occur within the shallow region below the surface, leading to crack propagation within a finite thin layer. The large temperature gradients induce large deformations between the surface and the interior and cause irreversible thermal damage. Once the damage accumulates up to a point, thermal fatigue cracks are produced. Figure 2(c) shows the distribution of the first principle stress along the depth direction. The tensile stress owing to the LCF thermal loading reaches maximum on the surface and decreases to zero at depth. On the surface, the compressive stress owing to the HCF thermal loading is the highest and decreases to zero below a specific depth. However, the interaction depth under the LCF thermal loading is slightly larger than that under the HCF thermal loading owing to the higher temperature range. In Figure 2(d), plastic stain accumulates within a finite small thickness under the LCF condition, whereas there is almost no plastic strain under the HCF condition. It is noteworthy that, during the CCF testing under variable amplitude loads, the direction of principal stress varies with cyclic loading and is time-dependent, but the amplitude of the principal stress may not be 
proportional. The above two factors suggest that the materials are under multiaxial thermal fatigue conditions, and the resulting CCF thermal cracks are supposedly different from those owing to uniaxial fatigue.

The surface crack patterns after thermal fatigue testing are shown in Figure 4. Compared to the LCF tests, besides several major cracks, more secondary cracks are observed in the CCF tests, causing denser crack networks. It is suggested that the transient temperature change is superimposed on the surface by the pulsed HCF thermal loading. The initiation of thermal cracks under variable amplitude thermal cycles is owing to tensile stress during the cooling stage and peak compressive-stress-induced cracking occurs during the highcycle stage. Because the HCF thermal loading promotes surface compressive cracking, accelerated crack propagation and higher crack density is expected. Owing to the interaction between thermal loadings under variable amplitude, the effect of superimposed HCF loadings on the growth of fatigue cracks is complex.

As mentioned above, the temperature difference caused by rapid cooling is large enough to produce plastic deformation, which is critical to crack initiation. Once the thermal cracks propagate in the depth direction, the deformation gradually becomes elastic. Finally, a fully elastic zone without tensile stress forms and no further propagation occurs. This is consistent with the fact that thermal cracks only occur within a thin layer near the specimen surface and crack growth along the depth direction is inhibited. Furthermore, the temperature change with high repetition rate on the surface produces cyclic elastic stresses under the yield limit, which is dynamic and has shorter interaction time and smaller interaction depth, when compared to the LCF condition. The HCF thermal loadings act on the initiated surface cracks in the wedging process and increase the crack tip opening displacement under high-frequency cyclic stresses, offering additional driving force to crack propagation. The wedging also enhances the inward transfer of oxygen, causing severe internal oxidation and crack-growth acceleration. Therefore, during the LCF test with superimposed HCF loading, the crack-growth rate with respect to the LCF cycle number is

$\left(\frac{\mathrm{d} a}{\mathrm{~d} N}\right)_{\mathrm{LCF}}=\mathrm{C}_{\mathrm{LCF}}\left(\Delta K_{\mathrm{LCF}}\right)^{m}+\sum_{i=0}^{N_{\mathrm{HCF}}} \mathrm{C}_{\mathrm{HCF}}\left(\Delta K_{\mathrm{HCF}}\right)^{m}$,

where $m$ and $C_{\mathrm{LCF}}$ and $C_{\mathrm{HCF}}$ are constants, $N_{\mathrm{HCF}}$ is the number of HCF cycles per LCF cycle, and $\Delta K_{\mathrm{LCF}}$ and $\Delta K_{\mathrm{HCF}}$ are the amplitudes of the stress intensity factor under LCF and HCF thermal loadings, respectively.

In this study, the thermal fatigue behavior under variable amplitude induced by the millisecond pulsed laser is investigated. The numerical results for different crack parameters agree with the experimental observations, verifying the accuracy of the proposed model. The observed cracks

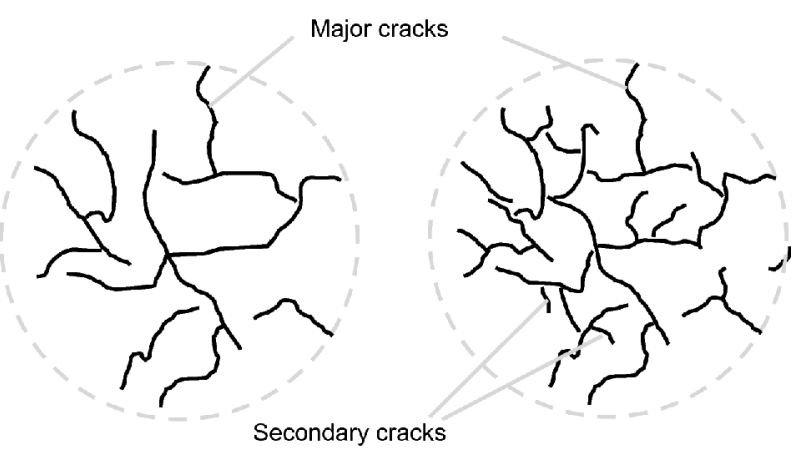

(a) LCF

(b) CCF

Figure 4 Schematic crack patterns on the surface: (a) LCF test; (b) CCF test.

with limited depth can be explained by the differences in temperature, stress, and plastic strain within a finite thin layer below the surface. The thermal fatigue behavior of cast iron under variable amplitude loading is modeled experimentally and numerically and is applicable to other materials under similar thermal conditions.

This work was supported by the National Natural Science Foundation of China (Grant Nos. 11272316, 11502269, 11672304, and 11272317), and the Instrument-Developing Project of the Chinese Academy of Sciences (Grant No. yz201636).

1 D. Zhu, and R. A. Miller, Surf. Coat. Tech. 108-109, 114 (1998).

2 M. Kutsuna, S. Fujita, Y. Sugita, and K. Yamad, in Interaction of Both Plasmas in $\mathrm{CO}_{2}$ Laser-MAG Hybrid Welding of Carbon Steel: LAMP 2002: International Congress on Laser Advanced Materials Processing, 3 March 2003, Osaka, Japan (SPIE, Osaka, 2003).

3 X. Z. Xiao, H. J. Chu, and H. L. Duan, Sci. China-Phys. Mech. Astron. 59, 664601 (2016).

4 X. Lu, Q. Li, W. Zhang, Y. Guo, T. He, and D. Zou, Appl. Thermal Eng. 50, 168 (2013).

5 Q. Zhang, Z. Zuo, and J. Liu, Eng. Fail. Anal. 34, 51 (2013).

6 X. Wang, and W. Zhang, Oxid. Met. 87, 179 (2017).

7 X. Wang, W. Zhang, B. Guo, and W. Zhao, Mater. Sci. Eng.-A 609, 310 (2014)

8 T. Tsuyoshi, and K. Sasaki, Procedia Eng. 2, 767 (2010).

9 X. H. Xu, S. L. Sheng, C. Tian, and W. J. Yuan, Sci. China-Phys. Mech. Astron. 59, 674611 (2016).

10 Z. Gan, G. Yu, S. Li, X. He, R. Chen, C. Zheng, and W. Ning, Chin. J. Aeronaut. 29, 1018 (2016).

11 G. Yu, X. He, and S. Li, Laser Manufacturing and Its Applications (National Defense Industry Press, Beijing, 2016), p. 96.

12 M. Schaus, and M. Pohl, Metall 52, 464 (1998).

13 M. Wang, Y. Li, Z. Wang, and E. Bao, J. Rare Earths 29, 489 (2011).

14 S. G. Long, and Y. C. Zhou, Compos. Sci. Tech. 65, 1391 (2005).

15 Y. Wang, N. Shen, G. K. Befekadu, and C. L. Pasiliao, Int. J. Heat Mass Transfer 113, 1246 (2017).

16 S. Pan, G. Yu, S. Li, X. He, C. Xia, W. Ning, and C. Zheng, Opt. Laser Tech. 99, 382 (2018).

17 S. Sihn, L. B. Childers, C. T. Walters, M. S. Forte, A. K. Roy, and J. P. Vernon, Int. J. Heat Mass Transfer 102, 1034 (2016).

18 D. Zhu, and R. A. Miller, Mater. Sci. Eng.-A 245, 212 (1998).

19 U. Grigull, and H. Sandner, Heat Conduction (Hemisphere Publishing Corporation, Washington DC, 1984), p. 136. 